\section{An analysis of the therapeutic benefits of genotyping in pediatric hematopoietic stem cell transplantation}

\author{
Felicity A Wright ${ }^{1,2,3}$, Mary Bebawy 3 Tracey A O'Brien ${ }^{*, 1,4}$
}

\begin{abstract}
Hematopoietic stem cell transplantation is a high-risk procedure that is offered, with curative intent, to patients with malignant and nonmalignant disease. The clinical benefits of personalization of therapy by genotyping have been demonstrated by the reduction in transplant related mortality from donor-recipient HLA matching. However, defining the relationship between genotype and transplant conditioning agents is yet to be translated into clinical practice. A number of the therapeutic agents used in stem cell transplant preparative regimens have pharmacokinetic parameters that predict benefit of incorporating pharmacogenomic data into dosing strategies. Busulfan, cyclophosphamide, thio-TEPA and etoposide have well-described drug metabolism pathways, however candidate gene studies have identified there is a gap in the identification of pharmacogenomic data that can be used to improve transplant outcomes. Incorporating pharmacogenomics into pharmacokinetic modeling may demonstrate the therapeutic benefits of genotyping in transplant preparative regimen agents.
\end{abstract}

The second diploid human genome to be sequenced and made publicly available and the first to be defined using next-generation sequencing belonged to James D Watson [1]. A biologist and a luminary of his generation, he was best known for his role in defining the structure of the DNA double helix. The publication of Watson's genome demonstrated that genomic technologies could be used to pursue personalized medicines theory. In his twilight years Watson required $\beta$-adrenergic antagonists to manage hypertension and described the sedative side effects experienced as leaving him "constantly falling asleep at inappropriate moments" [2].

$\beta$-adrenergic antagonists are metabolized by the hepatic micro-enzyme system and genetic variations in the gene that encodes for the CYP2D6 enzyme on chromosome 22 is associated with variability in drug response and in tolerability [3]. Watson's genome revealed he was homozygous for CYP2D6*10, that he had inherited two nonfunctional alleles which rendered him an intermediate metabolizer of this drug class. This insight into his own genetic code allowed Watson to reduce the $\beta$-blocker dosing frequency and ameliorate the malaise and drowsiness without compromising the antihypertensive effect [2].

Despite Watson's case, pharmacogenomic principles to guide dosage regimen design to an individual's genotype remain in the most instances theoretical. In cardiology, where $\beta$-blockers are prescribed most frequently, patients are not routinely genotyped and there is no wide spread genome led dosing for any of the other pharmacological classes used to manage hypertension [3].

'Kids Cancer Centre, Sydney Children's Hospital, Randwick, NSW, Australia

2Pharmacy Department, Prince of Wales \& Sydney Children's Hospitals, Randwick, NSW, Australia

${ }^{3}$ Graduate School of Health University of Technology Sydney, NSW, Australia

${ }^{4}$ School of Women \& Children's Health, University of New South Wales, NSW, Australia

*Author for correspondence: t.obrien@unsw.edu.au

\section{KEYWORDS}

- pediatrics

- pharmacogenetics

- polymorphism • stem

cell transplantation

- transplantation

conditioning 
In the most part this is due to lack of sufficient evidence to demonstrate a clear link between genotype and phenotype, as well as a multitude of alternative therapeutic options for treating hypertension. This vast list of antihypertensives includes agents that vary mechanistically, have a wide therapeutic margin, have diverse side effect profiles and hence it is simply easier, less time consuming and more cost effective to adopt empirical approaches in dosage individualization. In cancer medicine however, dosing strategies exist at the edge of patient tolerability; most agents have a narrow therapeutic window and in some treatment options such as in hematopoietic stem cell transplantation, the therapeutic index is intentionally exceeded in order to achieve myeloablation and allow engraftment of the donor marrow in the hematopoietic niche. Consequently genotype-based dosage individualization provides an opportunity to reduce toxicity and improve transplant outcomes.

\section{A short history of genotyping in stem cell transplant}

The improvement in overall survival in pediatric malignancies can be described as one of modern medicines true success stories. The application of multimodality treatment is credited with the improvement in overall survival, as is the enrollment of children on clinical trials [4]. Advances in treatment and also in diagnostics have seen overall survival increase and in some cases, the toxicities reduced. In some high-risk malignancies however, relapse remains a real risk and stem cell transplant is the only potential curative therapy [5]. In stem cell transplant patients the treatment toxicity burden is high and although improving, relapse and nonrelapse mortality along with acute and long-term toxicity remains a significant cause of concern [6]. This makes the process of genotyping as a clinical approach in the management of drug-related toxicity an attractive proposition.

The allogeneic transplant journey is divided into a number of overlapping phases (Figure 1). The first phase, the recipient donor search could be described as the poster child for the translation of genotyping into clinical practice [7]. High-resolution molecular typing of type I and type II HLA is employed in the donor-receipt search, and it has been demonstrated that there is a correlation between the number of mismatched alleles and the relative risk of mortality [8] with a fully matched donor recipient pair exhibiting lower rates of engraftment failure, acute and chronic graft versus host disease (GVHD) and transplant-related mortality when compared with mismatched donors [7]. The tolerance of disparity for each additional mismatch demonstrates that genotyping of the MHC; the most densely populated portion of the human genome can be used to improve transplant outcomes [7].

In contrast to the evolution seen in tissue typing procedures, the introduction of genetic or genome-based therapeutic strategies have not been seen in the antineoplastic agents used in transplant preparative regimens. The therapeutic approach has remained largely the same as in early transplant protocols and when a myeloablative regimen intensity is employed, the doses and pharmacological agents utilized are similar to those developed from early canine models [9] and tested in subsequent human trials [10]. This level of empiricism in the therapeutics of transplantation is explained by the relative high-risk nature of the procedure and the small numbers of patients transplanted each year comparative to other treatment modalities [6,11-12]. Clinical trials that result in evidence-based guidelines are limited and despite the precedence set by HLA typing utilizing genetic information to guide donor choice, drug dosing strategies that utilize germline genotypes are rare in the transplant discipline.

The lack of uptake of pharmacogenomics is evidenced by the current regulatory status of pharmacogenomic guided labeling of therapeutic substances by the US FDA. There is limited pharmacogenomic detail in the labeling of therapeutic substances with 170 individual drug listings on the FDA Table of Pharmacogenomic Biomarkers in Drug Labeling [13]. Out of the 37 agents on the list routinely used in cancer medicine, eight are conventional cytotoxic antineoplastic agents. The agents and their molecular targets are listed in Table 1. In addition to conventional antineoplastic agents, there are 11 monoclonal antibodies and 18 small molecules and other inhibitory molecules on the list, however the genomic information for these agents is related to specific actions of the agent based on tumor biomarkers.

Out of the eight cytotoxic chemotherapeutic agents in Table 1, four contain pharmacogenomic dosing information that is related to dosing strategy; cisplatin, irinotecan, mercaptopurine and thioguanine. When the FDA genotype labels are investigated for pharmacotherapeutic application in stem cell transplant, only one 


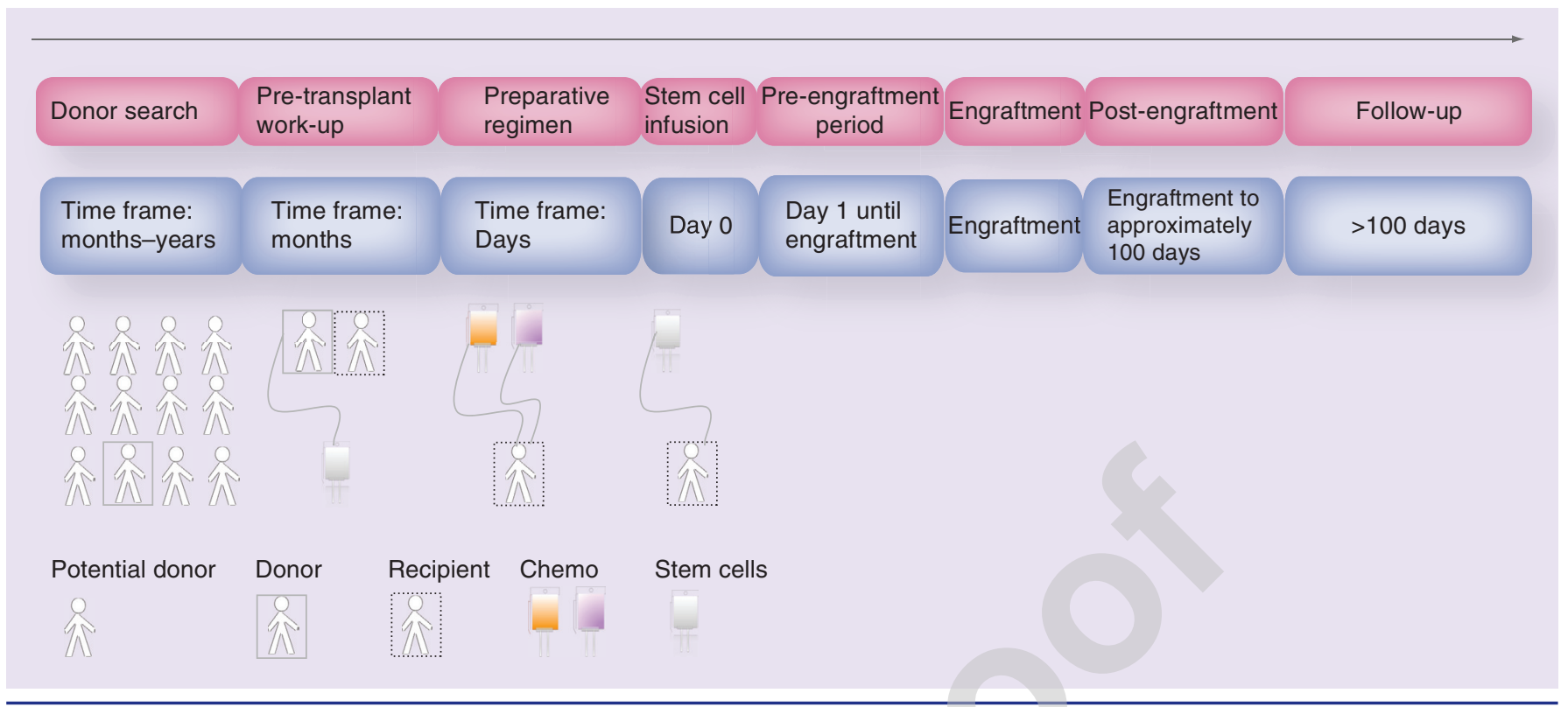

Figure 1. Allogeneic transplant journey stages.

chemotherapeutic agent is represented, busulfan. However, the specific details refer to a superseded oral tablet formulation label from 2003 that indicated the drug might be less effective in patients who expressed the Philadelphia chromosome [13]. This leaves no preparative regimen agents on the FDA list with pharmacogenomic information in the label.

There are a further three agents on the FDA list that would be considered part of the transplant formulary. Denileukin difitox is used in steroid refractory GVHD; however, it would not routinely be used in a first- or second-line setting [15]. The remaining two, trimethoprim/ sulfamethoxazole and voriconazole are used routinely as part of the prophylactic strategy for supportive care [16]. The information for trimethoprim/sulfamethoxazole refers to glucose6-phosphate dehydrogenase deficiency leaving only voriconazole as the only pharmaceutical agent used routinely in the transplant formulary with pharmacogenomic dosing information included on the label.

\section{Preparative regimen agent pharmacogenomics}

Due to highly toxic treatment regimens, the transplant community were early adopters of a personalized approach to care delivery in each phase of the transplant course (Figure 1). However more recent approaches to personalize therapeutics including pharmacogenomics have not been integrated, evidenced by the lack of pharmacogenomic data in FDA labeling and by gaps in the current published literature that tie pharmacogenomic information to improvement in transplant outcomes. The translational impact of pharmacogenomics has great potential and so far candidate gene studies have shown that genotype can be studied in the context of stem cell transplant preparative regimens; however, clinical relevance is yet to be elucidated.

Preparative regimen agents that cause full or partial myeloablation along with pharmacological immunosuppression are important causes of morbidities such as delayed engraftment and end-organ damage including acute kidney injury or hepatic dysfunction [17]. Pharmacogenomic principles examine the relationships among polymorphisms in drug metabolizing enzymes, drug pharmacokinetics and tumor response and treatment toxicity. There are a number of genes that encode for enzymes that hinder metabolism of preparative agents demonstrating that individual differences in drug efficacy or toxicity can be influenced by genetic factors [18].

There are a number of approaches used to identify which genes are responsible for variations in drug behavior [19]. Although it is expected that single nucleotide polymorphism (SNP) studies and genome-wide SNP arrays will provide further details, to date, in stem cell transplant the methodology used in the majority of studies are candidate gene in nature. The candidate gene approach has identified a number of transplant end points that correlate with 


\begin{tabular}{|c|c|c|c|}
\hline Drug & HUGO Symbol & Referenced subgroup & Action based on genotype \\
\hline Arsenic trioxide & $P M L / R A R A$ & $\begin{array}{l}P M L / R A R \alpha(\mathrm{t}[15 ; 17]) \text { gene expression } \\
\text { positive }\end{array}$ & Clinical indication \\
\hline Busulfan & Ph chromosome & Ph chromosome negative & $\begin{array}{l}\text { Refers to previous noncurrent label for tablet formulation } \\
\text { (2003) }\end{array}$ \\
\hline Capecitabine & DPYD & DPD deficient & Contraindication \\
\hline Cisplatin & TPMT & TPMT intermediate or poor metabolizers & Increased toxicity with $T P M T^{*} 3 B$ and $T P M T^{*} 3 C$ \\
\hline Fluorouracil & $D P Y D$ & DPD deficient & Contraindication \\
\hline Irinotecan & UGT1A1 & UGT1A $1 * 28$ allele carriers & $\begin{array}{l}\text { UGT1A1 7/7 genotype consider dose reduction. Increased } \\
\text { risk of neutropenia }\end{array}$ \\
\hline Mercaptopurine & TPMT & TPMT intermediate or poor metabolizers & Upfront dose reduction for homozygous-TPMT deficiency \\
\hline Thioguanine & TPMT & TPMT poor metabolizer & Upfront dose reduction for homozygous-TPMT deficiency \\
\hline
\end{tabular}

genotype. A correlation between suspected toxicities and individual agents may, in time, identify those at risk of adverse outcomes [17].

The hematopoietic stem cell transplant preparative or conditioning regimen consists of chemotherapeutic and immunological agents administered in adjunct with total body irradiation in certain patient cohorts. The regimen serves three main functions. To produce a profound immunosuppressive effect on the recipient to facilitate engraftment of donor marrow, to make space in the hematopoietic niche and, if the transplant is for a malignant indication, the combination of therapies aggressively treat the malignant disease [5].

The hallmark of antineoplastic therapy in stem cell transplantation is that the patients are treated beyond the limit of acceptable toxicity then rescued with hematopoietic stem cell reinfusion. The therapeutic index of the conditioning agents is intentionally exceeded in order to allow engraftment of recipient marrow and the resulting toxicity of these agents is thus a significant burden. Unlike many antineoplastic protocols where multiple agents are given over many months or even years the transplant preparative regimen sees all active agents given in a short space of time, generally in under 1 week. In addition much higher doses are generally applied. This potentially alters both the pharmacokinetic and pharmacodynamic properties of the agents and generates a high level of toxicity in the transplant recipient [5].

There is evidence at the receptor level that polymorphisms alter pharmacodynamic response and that variation in the genes that encode intracellular enzymes, cell surface receptors, transmembrane ion channels and drug transporters result in both expected adverse reactions, off-target toxicities and idiosyncratic effects [20]. In the stem cell transplant setting, there are a number of candidate gene studies that have attempted to correlate genotype with pharmacokinetic parameters. There are also a small number of studies that have investigated the association of common functional and nonfunctional alleles with transplant outcomes by preparative regimen agent. Busulfan and cyclophosphamide are the workhorses of the transplant preparative regimen formulary and hence their use is the most extensively studied.

\section{Busulfan}

Busulfan is a bifunctional alkylating agent [20] that is used as a preparative regimen agent in malignant and nonmalignant stem cell transplantation [21]. Its use has become more prevalent with the introduction in the late part of last century of an intravenous formulation, which offers a favorable and more predictable pharmacokinetic profile when compared with oral administration [22-24]. In addition the shift from oral to intravenous drug administration has been embraced due to the large number of tablets required when using the oral formulation.

For busulfan, like many other alkylating agents with steep dose-response curves, exposure predicts efficacy. Due to this dose-response relationship and inconsistent interpatient pharmacokinetic profiles, the majority of transplant centers employ therapeutic drug monitoring [25] with either area under concentration time curve (AUC) or concentration steady state measured with the first-administered dose. Subtherapeutic exposure on first dose pharmacokinetics either by AUC or concentration steady state predicts 
graft rejection and disease recurrence while supratherapeutic levels result in toxicity [21,25-36]. There is a link between high first dose exposure levels and endothelial damage resulting in transplant-related microangiopathy, sinusoidal obstructive syndrome or veno-occlusive disease (VOD) and capillary leak syndrome [26].

Current dosing strategies of pharmacokinetic guided and weight-adjusted dosing leave some patients outside the target therapeutic range during the first phase of the preparative regimen [27] and limitations in logistics prevent follow-up pharmacokinetic monitoring due to lag time in returning results [37]. This situation has resulted in a number of novel candidate gene studies being undertaken with the aim to better predict starting dosing requirements [25,27,30-34,38]. These studies, their gene targets and correlation between genotype and either pharmacokinetic or outcome data are presented in Table 2 .

The metabolism of busulfan lends itself to both pharmacokinetic and pharmacogenomic directed dosing. From a pharmacogenomic perspective there is a high level of polymorphism in the main isoform in the enzyme family responsible for busulfan metabolism and there is also a relationship between enzyme regulation and plasma concentrations of busulfan [21,28]. The alkylating moieties in busulfan undergo metabolic electrophilic biotransformation solely via glutathione conjugation [26] and this conjugation pathway is primarily catalysed by glutathione-Stransferase- $\alpha(G S T-A)$ [27,29]. Once conjugated, the alkylating capacity of busulfan is diminished.

In the hematopoietic stem cell transplant procedure busulfan produces dose-dependent myeloablation [5]. The hematopoietic stem cells in the bone marrow lack the gene to code for the GST-A enzyme and hence the alkylating capacity of busulfan is not reduced by the presence of glutathione-S-transferase. Like hematopoietic stem cells, endothelial cells either lack, or have reduced levels of the isoenzymes of GST and in particular sinusoidal cells lack the GST-A isoform [26]. On exposure to busulfan, reduced intracellular capacity for glutathione production in sinusoidal epithelial cells compared with hepatocytes results in extended busulfan activity and subsequent sinusoidal obstructive damage [27].

\section{Polymorphisms in glutathione-S-} transferase

The glutathione-S-transferase family are a group of enzymes involved in the phase II detoxification of a substantial number of therapeutic agents [18] and the cystolic isoenzymes in humans include GST-A, GST-M, GST-P and GST-T. GST-A has the strongest relationship with busulfan biotransformation where GST$A 1-1$ is the predominant isoform catalysing the conjugation of busulfan with glutathione [27]. Like the HLA genes that code for cell surface proteins used to match donor to recipient there is a high degree of allelic polymorphism in the glutathione family [29]. There is also an interdependence that exists in the gene expression and the subsequent effect on pharmacokinetic behavior. This makes the glutathione-S-transferase family an a priori candidate for pharmacogenetic studies of busulfan in stem cell transplant patients.

\section{Pharmacokinetics, transplant outcomes \& glutathione-S-transferase isoenzyme function \\ - GST-A1}

There are a number of studies that have evaluated the relationship between GST-A1 polymorphisms and busulfan pharmacokinetics in pediatrics [25,31], in adult transplant populations [33,38] and in both age cohorts [32] as well as GST-A1 polymorphisms and clinical outcomes using pharmacokinetic modeling in pediatrics [27,29-30] and in adults $[34,36]$.

In the pediatric studies that looked purely at pharmacokinetics, two studies independently demonstrated that there was a relationship between GST-A1 mediated drug clearance and patient genotype. Johnson showed that compared with $G S T-A 1{ }^{*} A /^{*} A$ wild-type, intravenous busulfan clearance was decreased by $30 \%$ for patients who were either hetero or homozygous variants $A 1{ }^{*} A{ }^{*} B$ or ${ }^{*} B /{ }^{*} B$ [25]. A relationship between enzyme expression and gene or promoter region mutations in linkage disequilibrium and clearance was also found [25]. Where mutations in the promoter regions of the GST$A 1$ gene $(-631 \mathrm{~T}>\mathrm{G},-567 \mathrm{~T}>\mathrm{G},-69 \mathrm{C}>\mathrm{T}$ and $-52 \mathrm{G}>\mathrm{A}$ ) were associated with reduced clearance [25]. A second study analyzing the pharmacokinetics of intravenous busulfan in patients with $\beta$-thalassemia major demonstrated a $10 \%$ reduction in clearance for $G S T-A 1^{*} B$ genotype patients compared with wild-type [31]. This was in contrast to an earlier study using oral busulfan in thalassemia patients where GST-A1 was associated with a lower $\mathrm{C}_{\max }$ and higher clearance [39]. It is unknown if the pharmacogenomic 


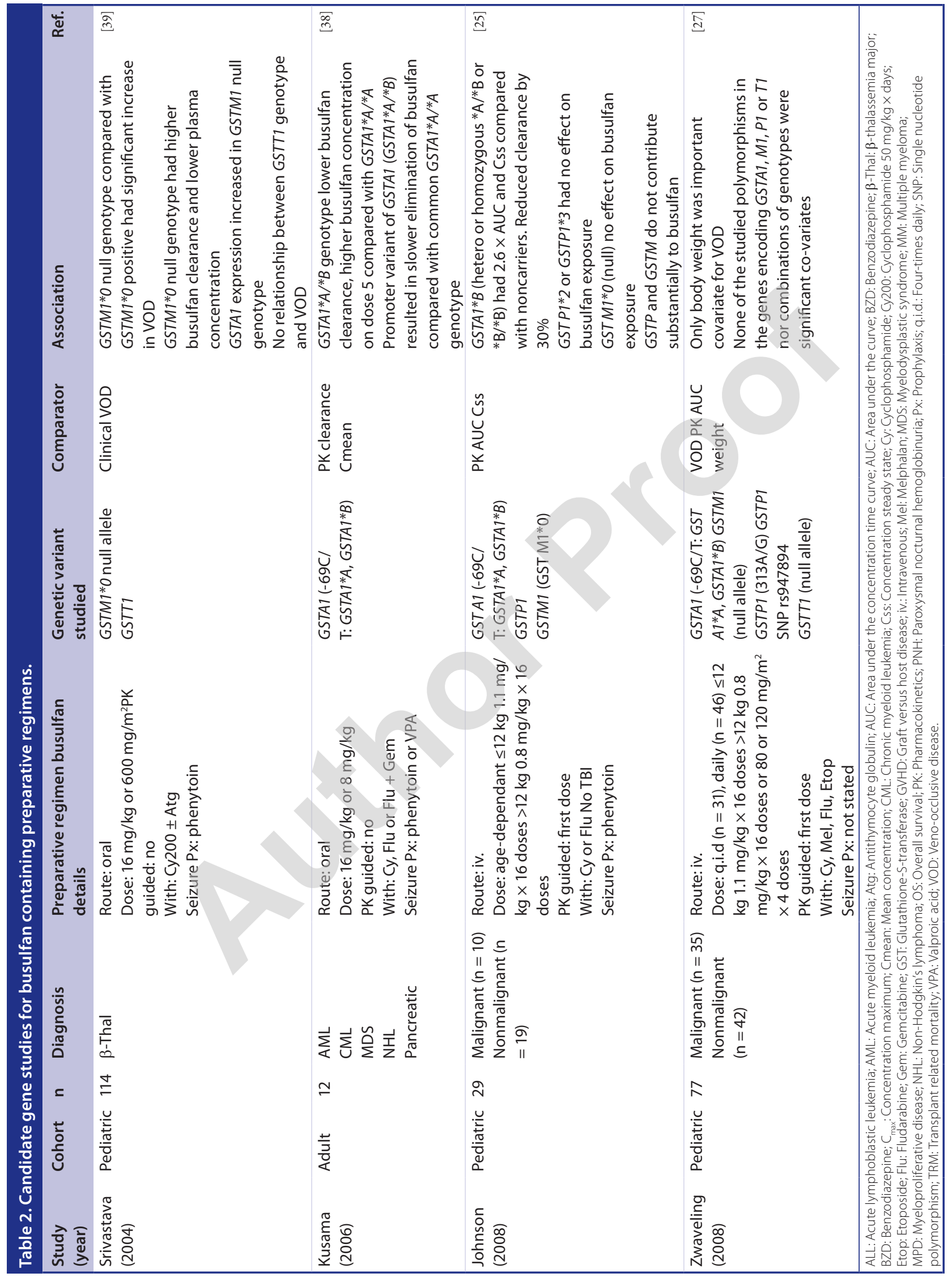




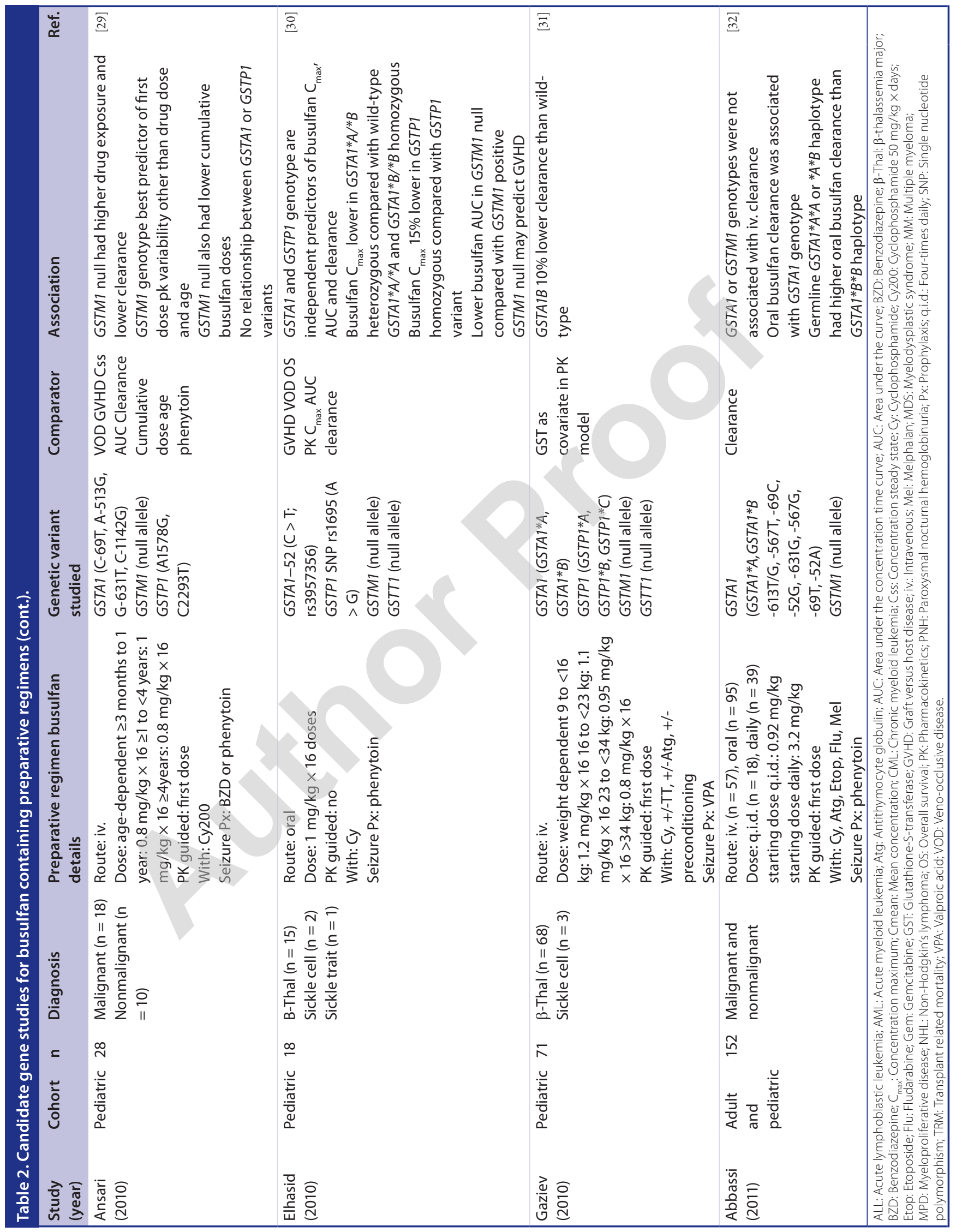




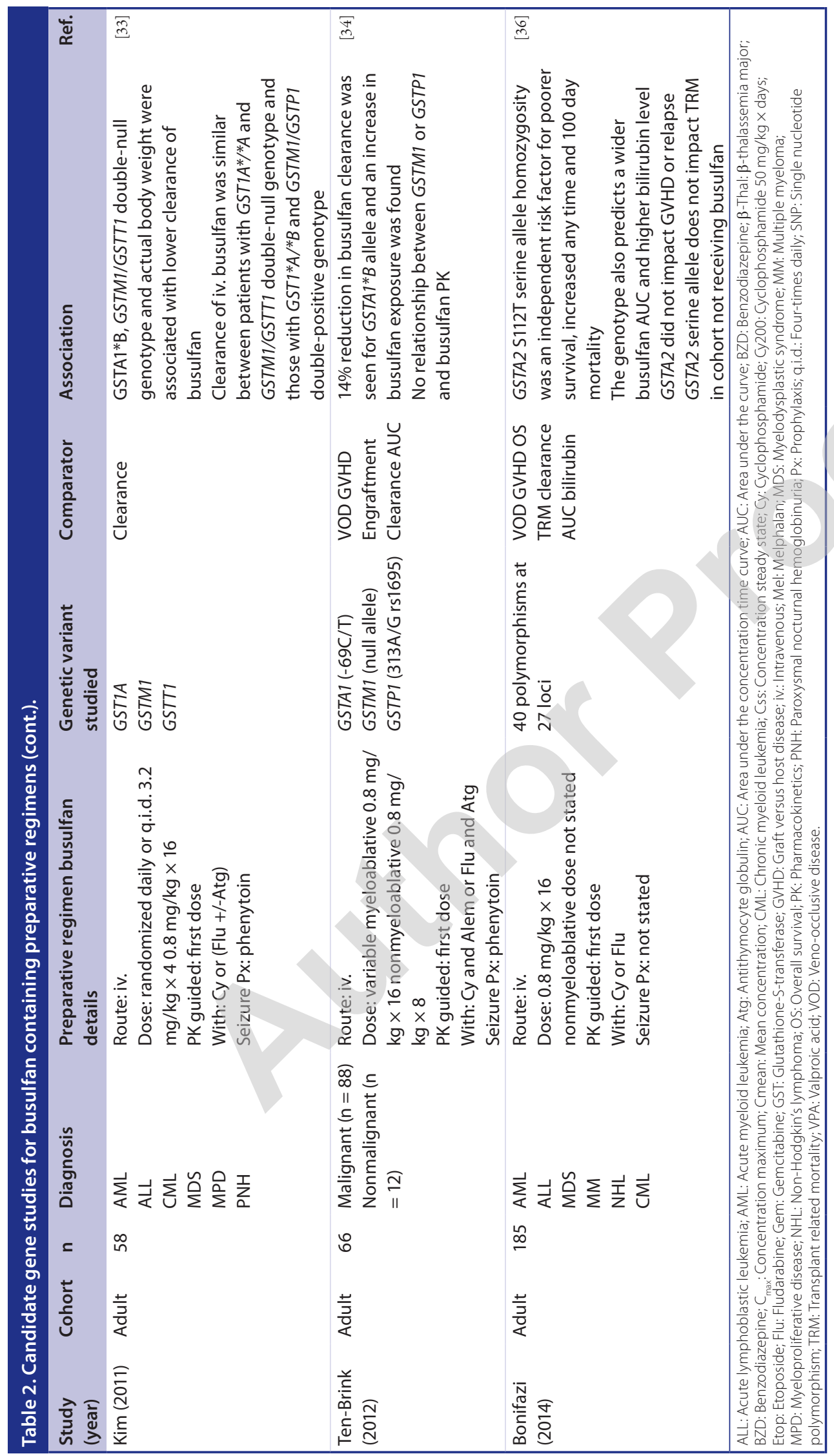


profiles and genotype correlation are similar for intravenous and oral busulfan preparations particularly in thalassemia patients who present for transplant with a high incidence iron overload and hepatic dysfunction [30].

There are three adult studies that are in concordance with these pediatric results. In an early study in 2006 Kusama showed that in 12 adult patients conditioned with oral busulfan without pharmacokinetic guided dosing, there was reduced clearance on the fifth dose in patients with the mutant allele $G S T-A 1^{*} A /^{*} B$ genotype compared with ${ }^{*} A /{ }^{*} A$ wild-type. It was demonstrated again, like in the Johnson study that it was the variant in the promoter region of GST$A 1\left(G S T-A 1^{*} A{ }^{*} B\right)$ that was responsible for the increased terminal elimination half-life of busulfan compared with $G S T-A 1^{*} A{ }^{*} A$ genotype [38]. Similarly a $14 \%$ reduction in busulfan clearance was seen for patients with the $G S T-A 1^{*} B$ allele and an increase in busulfan exposure was found in an adult study of 66 patients transplanted for malignant and nonmalignant disease [34].

In a sequence of studies Kim et al., first developed a multiplex PCR for seven genetic polymorphisms of GST [40] to allow high-throughput screening of GST alleles. This method was subsequently used in a clinical study, which demonstrated that GST gene polymorphisms significantly affected the pharmacokinetics of intravenous busulfan [33]. This group did not find a correlation for each individual isoform GST genotype but on multivariate analysis demonstrated that actual body weight and the combination of GST-A1*B and the GST-M1/GST-T1 double-null genotype was associated with lower clearance of busulfan [33].

For studies that assessed both clinical transplant outcomes and pharmacokinetic parameters in pediatrics, there was no relationship found between GST-A1 genotype [27,29-30]. There were correlations in transplant outcome data for another isoenzyme genotype, GST-M1-null however [29]. Regardless of the GST-A1 genotype, busulfan toxicity is increased in cells that have reduced intracellular production of GST; hence any allelic polymorphism that results in reduced enzyme function can theoretically lead to increased busulfan toxicity [26]. This may explain the delayed clearance, higher $\mathrm{C}_{\max }$ and AUC however the pharmacokinetic correlation doesn't translate to measurable clinical outcomes hence GST-A1 genotyping currently has an unknown role in clinical practice.

\section{- GST-P1}

The P1 isoform is the most abundant glutathione-S-transferase in pulmonary tissue and it has been demonstrated that SNPs in the polymorphic genes that encode the expression of enzyme results in reduced enzyme activity of GST-P1 [25]. However, this did not result in a correlation between genotype with pharmacokinetic or outcome end points in the majority of studies [25,27,29,33-34] although pulmonary dysfunction was not explicitly addressed as an assessed outcome factor. Only one study demonstrated a relationship between GST-P1 genotype and pharmacokinetic parameters for oral busulfan where $\mathrm{C}_{\max }$ was $15 \%$ lower in GST-P1 homozygous for the ancestral gene compared with GST-P1 variant SNP rs1695 [30]. It would be worthwhile to complete candidate gene studies that assessed the correlation between GST-P1 expression and interstitial pulmonary fibrosis associated with busulfan and pulmonary presentation of VOD.

\section{- GST-M1 \& T1}

Although the A1 isoform is the predominant contributor to busulfan biotransformation, GST$M 1$ and GST-T1 also contribute at half and a fifth of the activity of GST-A1 [27] where the contribution of GST-M1 is higher when GST$A 1$ activity is reduced [39]. Like the GST-A1 isoenzyme, GST-M1 and GST-T1 are highly polymorphic however unlike the A1 variant where polymorphism results in altered enzyme capacity, for the M1 and T1 isoforms polymorphisms results in a lack of protein product production and enzyme activity [29]. This results in a null genotype, $G S T-M 1^{*} 0$ or $G S T-T 1^{*} 0$ for individuals with homozygous deletion of the gene [25]. For the M1 isoform there are a number of studies that indicate that there is no relationship between pharmacokinetic parameters, clinical end points and the null genotype [25,27,32,34]. However, there are three studies that showed a relationship between the M1 null genotype and VOD [39], GVHD [30] and pharmacokinetic end points [29].

The two studies that demonstrated a relationship for transplant outcomes used oral busulfan without pharmacokinetic guided dosing in patients with thalassemia major or congenital hemoglobinopathies. In the first, 114 pediatric patients with thalassemia major receiving myeloablative doses of busulfan combined with cyclophosphamide were genotyped from 
peripheral blood prior to transplant [39]. Patients with homozygous deletion of GST-M1, the $G S T-M 1^{*} 0$-null genotype, had higher busulfan clearance and lower plasma concentration and an increase in incidence and severity of VOD compared with $G S T-M 1^{*} 0$ positive patients [39]. In the second oral study, 18 pediatric patients transplanted for thalassemia major, sickle cell or presence of sickle cell trait demonstrated that in patients with a $G S T-M 1^{*} 0$-null genotype had a lower busulfan AUC compared with positive genotype and that the presence of GST-M1-null may predict GVHD [30]. The results of these oral studies are confounded by the formulation of busulfan administered, lack of pharmacokinetic guided dosing and the presence of liver dysfunction in the patients at the time of presentation for transplant [30]. In the study that used intravenous busulfan with first dose pharmacokinetic guided dosing no relationship with clinical outcomes was found [29]. However, from a pharmacokinetic perspective the GST-M1 null genotype was associated with higher drug exposure, lower clearance and a lower cumulative dose. This study also found that other than drug dose and age, GST-M1 genotype was predictor of variability in first dose pharmacokinetic results [29].

GST-T1 does not contribute on a significant scale to the electrophilic biotransformation of busulfan $[30,32,35]$. Not surprisingly, GST-T1 has not been shown to be an independent variable in busulfan pharmacokinetic behavior in stem cell transplant patients. Its contribution to the overall pharmacokinetic profile is however demonstrated in a single study where presence of the GST-T1 null genotype in combination with the GST-M1 null genotype in patients who also exhibit $G S T-A 1^{*} B{ }^{*} B$ results in a lower clearance of intravenous busulfan in adult patients transplanted for malignant and nonmalignant diseases [33].

\section{- GST genotype conclusions}

Glutathione-S-transferase is the enzyme responsible for biotransformation of busulfan and glutathione conjugation by the GST isoenzyme family and is the sole mechanism by which busulfan undergoes this transformation [27]. Polymorphisms in the genes that encode the GST isoenzyme family result in differing levels or absence of expression and subsequently affect drug metabolism. In isolation it would be expected that activity of this isoenzyme family would be predictive of measurable pharmacokinetic parameters. However there is no clear relationship shown from multiple patient cohorts between hepatic enzyme gene expression, pharmacokinetic parameters and transplant outcomes based on GST genotype.

In addition, it must be remembered that no agent in transplant is administered in isolation and the presence of other agents in the preparative regimens as well as the supportive care used to prevent transplant related complications may influence the relationship between drug behavior and genotype. Busulfan lowers the seizure threshold and without prophylaxis tonic-clonic seizure can occur [41]. The majority of studies that assess the pharmacokinetics of busulfan use phenytoin as seizure prophylaxis. As phenytoin is a potent inhibitor of GST-A1 expression [28] in preparative regimens containing busulfan the role of the agent used for seizure prophylaxis must be considered. Ansari et al. included the effect of phenytoin in their analysis [29] and demonstrated that while co-administration of phenytoin was not significantly associated with busulfan pharmacokinetics or on multivariate analysis, with patient genotype, it would be prudent to include the presence of phenytoin in any future studies.

There is currently insufficient evidence to support the use of GST genotyping to guide busulfan dosing and predict pharmacokinetic parameters in hematopoietic stem cell transplantation. There is also insufficient evidence to implement pre-emptive supportive care based on GST genotype to prevent transplant related complications of GVHD and VOD.

\section{Nonbusulfan preparative regimen agents}

To carry out the main functions of the preparative regimen, immunosuppression, making space in the hematopoietic niche and treating the underlying malignancy, combination therapy is required. Alone, each agent would not produce the appropriate amount of ablation of marrow or prevent rejection in the transplant recipient. A wide range of therapeutic agents with nonoverlapping toxicities with or without total body irradiation are employed in the transplant preparative formulary resulting in a regimen of varying intensity and toxicity. The combination used is chosen by underlying disease, performance scores and donor source [5]. While the majority of published data attempting to correlate genotype with clinical outcome has been for busulfan, there are some key studies in other 
preparative regimen agents that include promising results for pharmacogenetic guided dosing. These studies are summarized in Table 3.

\section{Cyclophosphamide}

Like busulfan and other alkylators, cyclophosphamide has a profound dose-response curve [20]. It is most frequently used in a high dose in myeloablative regimens $[18,35,42]$. It has also garnered recent attention as re-emergence of its role post stem cell infusion to prevent GVHD [46]. The metabolism of cyclophosphamide is complex and produces active metabolites that facilitate engraftment and inactive metabolites that are implicated in transplant regimen toxicity [18]. Cyclophosphamide is a prodrug that is activated via hydroxylation in the liver predominantly by CYP2B6 although other members of the hepatic microenyzme family contribute with CYP2C19, CYP2C9 and CYP3A4/5 also involved in the metabolic process. The product of hydroxylation is 4-hydroxycyclophosphamide and this compound exists in tautomeric equilibrium with aldophosphamide $[47,48]$. When in the aldophosphamide form the compound decomposes to phosphoramide mustard, the active alkylating agent and acrolein, the metabolite responsible for endothelial toxicity. Minor pathways simultaneously generate metabolites, for example, chloroacetaldehyde the metabolite implicated in neurotoxicity is produced via CYP3A 4/5 mediated dechloroacetylation [48]. Cyclophosphamide is a component of most hepatotoxic myeloablative regimens and it is its activity on sinusoidal endothelial cells that results in liver microcirculation damage. Interpatient variability in levels of each metabolite are implicated in the extent of end organ damage post-transplant [47]. Due to the heavy dependency on hepatic hydroxylation, a large interpatient variability in the metabolic pathway and its contribution to transplantrelated toxicity cyclophosphamide is a good candidate for pharmacogenomic studies.

There are two key studies of cyclophosphamide use and patient genotype in hematopoietic stem cell transplant patients. In the first, Kim et al., studied 21 adult patients with thalassemia major who received cyclophosphamide and busulfan preparative regimen underwent genomic DNA analysis on pretransplantation peripheral blood [42]. Four SNPs in the drug transport enzymes, ATP-binding cassette subfamily B and C as well as eight SNPs and two null alleles in the hepatic micro enzyme family and glutathione-S-transferase were analysed. Inducible and noninducible clearance of cyclophosphamide and its relationship to the presence of the SNPs or null alleles was calculated using a hypothetical enzyme compartment model. $A B C C 2$ or multidrug resistance-associated protein 21249 genotype affected both noninduced and induced clearance of cyclophosphamide. In patients with the $A B C C 21249 \mathrm{GA}$ heterozygous genotype, noninduced clearance of cyclophosphamide was reduced by $51 \%$ compared with 1249GG wild-type. No other allelic variation was associated with clearance [42].

Rocha et al. examined adult and pediatric patients undergoing HLA-identical sibling transplant for de novo acute or chronic leukemia between 1994 and 1999. Although the study did not specifically look at cyclophosphamide, the drug was a component of the majority of preparative regimens (88/107). A correlation between a number of transplant outcomes and genotype was found. The findings included an association between toxicity and recipient genotype, with oral mucositis being associated with CYP2B $6 * 4$, hemorrhagic cystitis associated with recipient CYP2B6*2A, GVHD associated with recipient methylenetetrahydrofolate reductase (MTHFR) c677T and higher mortality and lower overall survival for recipients with vitamin $\mathrm{D}$ receptor Taql SNP. There were also some associations for donor genotype with chronic GVHD associated with donor GST-P1 and VOD associated with donor CYP2B6*6. There was no association of GST-P1, T1 or $M 1$ polymorphisms with liver toxicity of VOD [18].

There are a number of studies that have determined a correlation between cyclophosphamide and genotype in the published literature. The majority are in medical oncology, with breast cancer the most common pathology represented when the literature is reviewed. The literature in hematopoietic stem cell transplantation is more limited however large-scale studies using genomic array analysis are required to further understand the relationship between cyclophosphamide, donor and recipient genotype in the stem cell transplant discipline.

\section{Thio-TEPA}

Triethylenethiophosphoramide or thio-TEPA is a polyfunctional alkylating agent that rapidly undergoes CYP2B6 and 3A4 mediated oxidative desulfuration to TEPA [45]. Both thio-TEPA and TEPA are active alkylators [20] crosslinking 


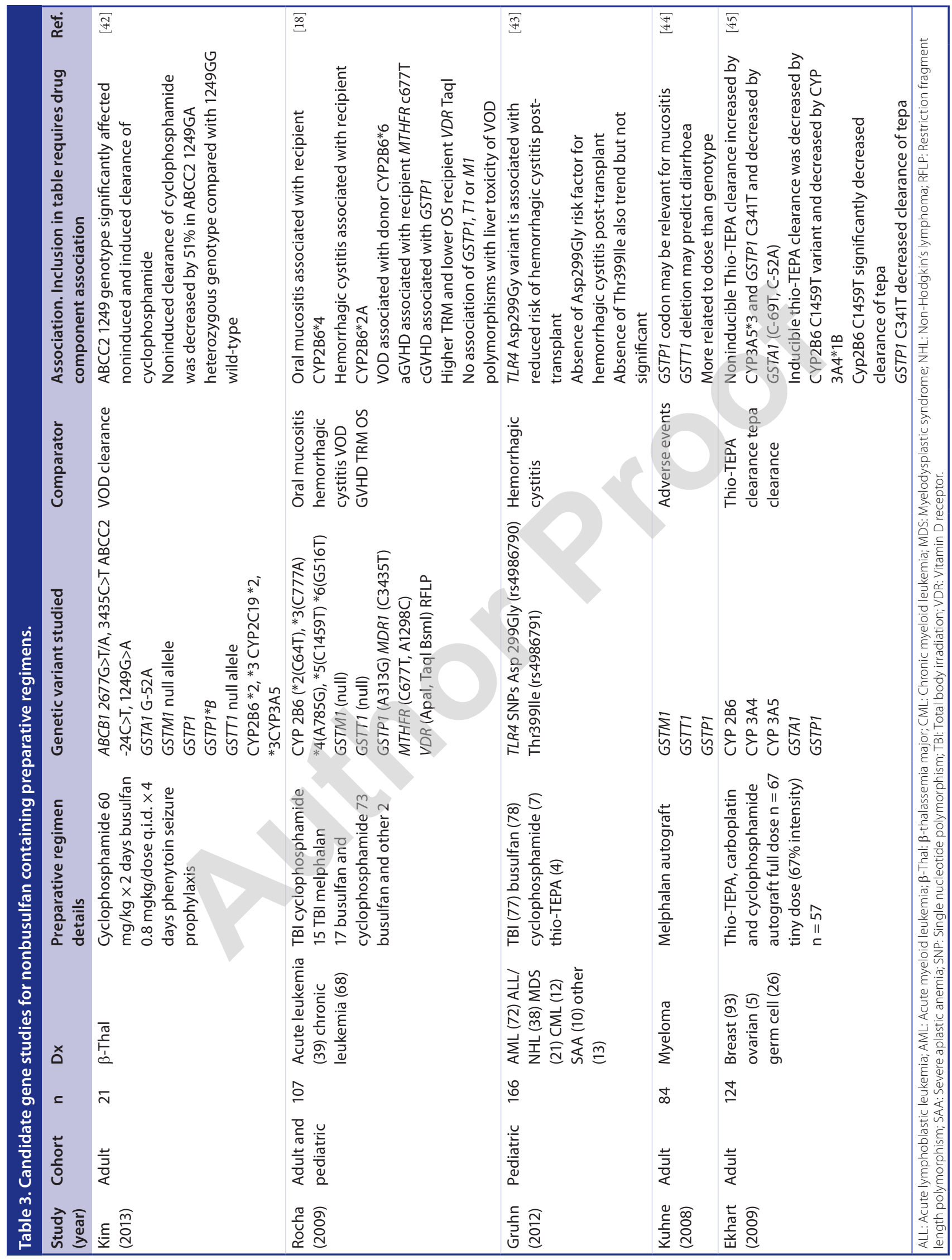


DNA strands by reacting with phosphate groups to inhibit DNA and RNA synthesis. In addition when combined with total body irradiation, thioTEPA has a radiomimetic action causing further disruption the bonds of DNA. There is a wide range of interpatient variability in the production of TEPA from thio-TEPA as well an inducible metabolic process in the presence of other drugs including cyclophosphamide [26]. This leads to an unpredictable efficacy and adverse effect profile [45]. In addition to the hepatic P450 isoenzyme desulfuration thio-TEPA and TEPA are conjugated to glutathione catalysed by GST A1-1 and P1-1 [45] thus mechanistically the drug is a candidate for pharmacogenomic guided dosing.

There is a single study that investigated the relationship between genotype and pharmacokinetic parameters in autograft patients receiving high-dose cyclophosphamide, thio-TEPA and carboplatin for breast cancer, ovarian and germ cell tumors [45]. Patients were genotyped for SNPs with expected association with thioTEPA metabolism and those with an allelic frequency of greater than $4 \%$ were included [45]. It was demonstrated that noninducible thio-TEPA clearance was increased in patients with the CYP3A5*3 and GST-P1 C341T genotypes and decreased in GST-A1 (C-69T, C-52A). Inducible thio-TEPA clearance was decreased by CYP2B6 $\mathrm{C} 1459 \mathrm{~T}$ variant and in those expressing the CYP $3 \mathrm{~A} 4^{*} 1 \mathrm{~B}$ genotype. For the TEPA metabolite, the presence of either CYP 2B6 C1459T SNP or GSP-P1 C341T SNP was associated with a decreased clearance of TEPA when compared with wild-type. CYP 2B6, CYP 3A4, CYP 3A5, GST-A1 and GST-P1 polymorphisms may contribute to clinical efficacy of thio-TEPA [45]. Genotyping patients receiving thio-TEPA is a promising area of research for stem cell transplant patients and warrants further candidate gene and genome array studies to determine if the autograft data can be translated into the allograft population.

\section{Etoposide}

There are no specific studies outlining the influence of genotype on etoposide efficacy or toxicity in hematopoietic stem cell transplant however as the agent is widely used in nontransplant medicine and is a substrate for multidrug resistance protein 1, CYP 3A4 and 5 and GST [49] there is thought that genotype may play a role in future dosing strategies. There are two key genotype studies that may guide future transplant trials. In the first study, genome wide methodology identified 63 SNPs associated with gene expression and etoposide-induced toxicity [50]. In the second, a nonparametric whole genome linkage analysis of five phenotypes, 22 SNPs on four genes were identified in playing a role in etoposide mediated topoisomerase inhibition and gene expression [51]. There are no studies investigating SNPs and clinical outcomes and to date the genome wide association studies have not translated into clinical practice. The role of genotyping patients receiving etoposide as part of their preparative regimen is still to be defined.

\section{Melphalan}

Melphalan is an alkylating agent that produces dose-dependent myelosuppression. Used frequently in the autologous and allogeneic stem cell transplant setting, the toxicities are known to be related to dose administered. There is one adult study published that analysed the relationship between GST-M1, GST-TI and GST-P1 and melphalan adverse events in patients receiving autograft for multiple myeloma [44]. The findings included that the GST-P1 codon may be relevant for mucositis and that GST-T1 deletion may predict diarrhea. However, adverse events were more like to be associated with dose than genotype [44] and no specific model for predicting adverse effects was generated.

There are no pediatric specific studies that analyse genotype with melphalan efficacy or toxicity in either the autograft or allograft setting. There were a small number of patients (17) in the Rocha study that received busulfan-melphalan regimen however there is no breakdown of patient characteristics by preparative regimen nor is it known many pediatric patients were in the busulfan-melphalan cohort.

\section{Fludarabine}

Fludarabine is a nucleoside analog that primarily provides dose-dependent immunosuppression in the preparative regimen. Widely applied in both myeloablative and nonmyeloablative regimens, the drug is characterized by its dose-dependent renal clearance and its role in neurotoxicity in the transplant recipient [5]. There are no studies regarding genotype and fludarabine in the transplant population however there is some promising data for the use of other nucleoside analogs in other specialities. In patients receiving nucleoside reverse transcriptase inhibitors for 
treatment for HIV, mitochondrial DNA polymerase polymorphisms have been indicated in the toxicity of these agents. As mitochondrial DNA polymerase gamma is the sole polymerase responsible for replication of the mitochondrial genome, polymorphisms may explain the wide range of toxicity and tolerability exhibited by these agents [52]. There is also evidence in chronic leukemia patients that fludarabine drug efficacy is related to variants gene expression [53]. There is promise that genotyping may help explain the variable pharmacokinetic and pharmacodynamic profile of fludarabine in transplant patients. Further candidate gene or SNP array studies are required to determine the significance of mitochondrial genotype and fludarabine efficacy in hematopoietic stem cell recipients.

\section{Pharmacological T-cell depletion}

Alemtuzumab and antithymocyte globulin are both used for pharmacological in vivo T-cell depletion in nonmatched sibling donor transplants [5]. Currently there are no pharmacogenomic studies that have investigated any correlation between genotype, pharmacokinetic parameters and transplant outcomes. The dissolution, metabolism and excretion pathways of antithymocyte globulins have not been the subject of pharmacogenetic analysis. There is a complex relationship between drug exposure and lymphocyte binding that is dependent on the individual's ability to metabolize foreign immunoglobulins. There is a potential that genotype may play a role in the T-cell depletion however this is yet to be elucidated.

For alemtuzumab the picture is less clear again, there is wide range of interpatient variability, the drug exhibits nonlinear time and white cell count dependent pharmacokinetics [54] however as the majority of pharmacokinetic studies come from its use in chronic lymphocytic leukemia it is difficult to extrapolate these results to the transplant setting. There is some work on individualizing immunosuppression including alemtuzumab in transplant patients however this has focused more on calcineurin and mammalian target of rapamicin inhibitors post stem cell infusion than preparative regimen T-cell suppression.

\section{GVHD prophylaxis \& treatment}

There is a small but significant set of publications regarding the relationship between genotype and efficacy and toxicity of post transplantation immunosuppression in the allogeneic setting. High-resolution molecular HLA typing does not completely ameliorate GVHD and it remains the leading cause of mortality in many transplant types [17] hence improvements would be welcomed. The pathophysiology of GVHD is complex and incompletely understood and although there is evidence that there is a high degree of allelic polymorphism in the immunomodulatory genes that govern GVHD there is no clear link between genotype and outcome.

Pharmacological immunosuppression posttransplant is one of the most difficult to categorize due to pharmacodynamic noise of dose adjustment based on drug levels. For cyclosporin [55] and mycophenolate mofetil [56] there are some key studies that associate genotype to GVHD incidence however there are no good models to support genotype led dosing.

There is literature surrounding the key polymorphisms in methotrexate metabolism and its application in GVHD prophylaxis. Short course methotrexate plus calcineurin inhibitor is the most widely used GVHD prophylaxis regimen in T-cell replete grafts and there have been a number of population pharmacokinetic models that have demonstrated a link between gene expression and methotrexate clearance [57-59]. In one study, Kim utilized a two-compartment model to demonstrate that $A B C-B 1 \mathrm{C} 3435 \mathrm{~T}$ SNP is associated with methotrexate clearance [60]. In patients with the $\mathrm{CC}$ or $\mathrm{CT}$ genotype clearance was increased by $21 \%$ compared with the TT genotype [60].

Other studies have reviewed the role of MTHFR the key regulatory enzyme in folate metabolism and methotrexate exposure and efficacy. In patients with GVHD two nonsynonymous SNPs in MTHFR (C677T and A1298C) influenced MTHFR activity [17] and resulted in an altered risk of GVHD. It is postulated that there is an interrelationship between MTHFR C677T and thymidylate synthase resulting in a reduced risk of GVHD due to greater methotrexate exposure associated with these alleles. It was also found that the MTHFR C667T SNP had a role in GVHD outcomes [58,59] where a reduced risk GVHD was seen possibly due to greater sensitivity to methotrexate. The role of genotyping patients in patients planned to receive short course methotrexate warrants further investigation. 


\section{Supportive care}

Currently donor and recipient serology and the presence of pretransplant infection determines anti-infective supportive care prophylaxis, however there are some novel studies that have analyzed the relationship between genotype and post-transplant infective complications [8]. There is a relationship between SNP haplotype and the incidence of gram-negative bacteria with a mutation in the promoter region of the lipopolysaccharide gene resulting in unchecked replication in patients with a rare homozygous variant [8]. There is also an association between SNP polymorphisms in toll-like receptor 4 and the presence of hemorrhagic cystitis where the incidence was $0 \%$ in pediatric transplant patients with a certain SNP haplotype [43]. As the pathogenesis of hemorrhagic cystitis is often related to common post transplant viral infections due to BK virus or adenovirus [43] as well as the acrolein metabolite of cyclophosphamide [18] this is certainly an area that warrants further investigation.

Also of note in the supportive care setting is the relationship between genotype and incidence of fungal infection and the efficacy of azole antifungals used to treat and provide prophylaxis against invasive infections [8]. It has been demonstrated that non-HLA polymorphisms are involved in pulmonary infections and that polymorphisms again in Toll-like receptor 4 result in an increase in the risk of invasive aspergillus infection after allogeneic stem cell transplantation [8]. In addition, polymorphisms in genes that encode the enzymes in common drug metabolism pathways, for example, GST and in hepatic isoenzyme P450 families result in variation in the measured pharmacokinetic parameters of azole antifungals [16]. As commercial availability of genotyping for these CYPs is available and the FDA includes guidance on allelic polymorphisms on the voriconazole label, this is perhaps the closest representation of pharmacogenomic guided dosing in clinical practice in hematopoietic stem cell transplantation.

\section{Conclusion}

Despite advances in chemotherapy and the advent of molecular targeted agents, allogeneic hematopoietic stem cell transplantation remains the only curative regimen for many high-risk hematological malignancies [5]. Overall survival has increased [5,11], due to a number of factors notably the use of high-resolution molecular typing to match the donor to the recipient as well as other factors such as earlier patient referral and improvements in supportive and psychosocial care $[4-5,17]$.

A range of studies, all of candidate gene design, have been published for busulfan and initial links between pharmacokinetic parameters, transplant related outcomes and overall survival are evident. The first exploratory studies, building on the candidate gene approach, have been published for busulfan. [6]] The exploratory methodology clinical application did not yield identifiable targets for busulfan and requires larger cohort studies to determine the clinical application. For cyclophosphamide the majority of evidence for pharmacogenomic behavior comes from nontransplant disciplines however there is one key study that has elucidated a relationship between cytochrome P450 polymorphisms and drug tolerability. The extension of autograft data shows promise in Thio-TEPA containing regimens and etoposide, has some preclinical data in laboratory cell lines but no clinical studies in transplant populations. There

Table 4. Transplant preparative agents with correlation between pharmacokinetics and candidate gene studies.

\begin{tabular}{|c|c|}
\hline Drug & Pharmacokinetic model correlation with genomic profile \\
\hline Alemtuzumab & $\begin{array}{l}\text { No model. Complex elimination kinetics dependent on white cell surface CD52 receptor expression prevents } \\
\text { correlation between genotype and pharmacokinetics }\end{array}$ \\
\hline Atg-equine & No model. Genomic profile unable to predict pharmacokinetic parameters due to lymphocyte binding \\
\hline Atg-rabbit & No model. Genomic profile unable to predict pharmacokinetic parameters due to lymphocyte binding \\
\hline Busulfan & Yes \\
\hline Cyclophosphamide & Yes \\
\hline Etoposide & Yes \\
\hline Fludarabine & Yes \\
\hline Melphalan & No model. Pharmacogenomic results explained by dose variance due to weight-based dosing \\
\hline Thio-TEPA & Yes \\
\hline Treosulfan & Insufficient data \\
\hline
\end{tabular}


Table 5. Future targets for preparative regimen agents where pharmacokinetic parameters correlate with genomic profile.

\begin{tabular}{ll} 
Drug & Candidate gene identified \\
Busulfan & GST family \\
\hline Cyclophosphamide & CYP2B6, GST-P1 \\
Etoposide & MRP1, CYP3A4/5, GST family \\
\hline Fludarabine & Mitochondrial DNA polymerase polymorphisms \\
\hline Thio-TEPA & CYP2B6, CYP3A4/5, GST-A1, GST-P1 \\
\hline GST: Glutathione-S-transferase. & \\
\hline
\end{tabular}

are limited data in other agents used frequently in preparative regimens including pharmacological T-cell depletion with antithymocyte globulin or alemtuzumab. Other notable exclusions from literature include fludarabine, melphalan outside the myeloma population and treosulfan.

Many therapeutic agents used in hematopoietic stem cell preparative regimens have pharmacokinetic and pharmacodynamic profiles that predict a theoretical benefit from genomic-based dosing. Despite this, and the extensive availability of genetic data and well-described drug metabolism pathways, genotype lead dosing has not been widely adapted. Current studies are limited to a candidate gene single conditioning agent structure and do not adequately explain the effect of other conditioning agents, posttransplant immunosuppression and the effect of the underlying disease or even the graft itself.

\section{Future perspective}

To date, in hematopoietic stem cell transplantation, pharmacogenomics have not had widespread translation to the bedside nor the clinic. Population genetics and pharmacokinetic modeling do not explain drug efficacy or toxicity primarily due to a lack of correlation between genotype and phenotype and the influence of environmental and epigenetic factors. The genotype-phenotype conundrum and the unknown effect of epigenetic factors currently prevents the widespread therapeutic application of genotype guided dosing in the hematopoietic stem cell transplant discipline.

In order to translate current pharmacogenomic knowledge into clinical practice, future research requires focus to be not only on the collection of genomic data for both patients and healthy controls but also on bioinformatics and the development of biostatical and pharmacokinetic models. There are a limited number of known correlations between pharmacokinetic and pharmacogenomic parameters for common preparative regimen agents (Table 4). Further exploration of the relationship between pharmacokinetic behavior and genotype for busulfan, cyclophosphamide, etoposide, fludarabine and thio-TEPA is warranted and may yield a therapeutic benefit for transplant patients. Potential targets for pharmacokinetic modeling at an individual patient or population level that include genomic data for these agents are shown in Table 5.

Although analysis of the genotype-kinetic relationship in order to develop dosing algorithms may allow a patient's pharmacogenomic profile to act as an adjunct to weight-based dosing and therapeutic drug monitoring, it must also be considered that non-HLA genotyping may have no role in predicting doses for hematopoietic stem cell transplant patients and that weight, surface area and where appropriate pharmacokinetic guided dosing are adequate to define dose.

Individualized dosing strategies in the highrisk procedure of hematopoietic stem cell transplantation are potentially of great significance to the transplant community. Genome wide or other novel methodologies that expand the knowledge of pharmacogenomics based on the candidate gene approach will assist in determining the future role of non-HLA genotyping in hematopoietic stem cell discipline. Future research identifying the relationship between pharmacokinetic behavior, pharmacodynamic response, genotype and phenotype will allow the clinical significance of non-HLA genotyping to be elucidated in the allogeneic transplant setting.

\section{Financial \& competing interests disclosure}

The authors have no relevant affiliations or financial involvement with any organization or entity with a financial interest in or financial conflict with the subject matter or materials discussed in the manuscript. This includes employment, consultancies, honoraria, stock ownership or options, expert testimony, grants or patents received or pending or royalties.

No writing assistance was utilized in the production of this manuscript. 


\section{EXECUTIVE SUMMARY}

- The hematopoietic stem cell transplant discipline was an early adopter of the principles of personalized medicine utilizing HLA genotyping to identify suitable transplant donors.

- High resolution molecular typing of HLA genotype has led to a significant improvement in overall survival and transplant-related outcomes.

- Overall survival, transplant-related mortality and drug toxicity from preparative regimen agents may have a relationship with genotype for some preparative regimen agents.

- Expansion of candidate gene methodology and studies that are able to correlate genotype and phenotype will define the future of the therapeutic benefits of genotyping in the hematopoietic stem cell transplant procedure.

\section{References}

Papers of special note have been highlighted as:

- of interest; $\bullet \bullet$ of considerable interest

1 Wheeler DA, Srinivasan M, Egholm M et al. The complete genome of an individual by massively parallel DNA sequencing. Nature 452(7189), 872-876 (2008).

2 Watson JD. Living with my personal genome. Pers. Med. 6(6), 607 (2009).

3 Bijl MJ, Visser LE, Van Schaik RH et al. Genetic variation in the CYP2D6 gene is associated with a lower heart rate and blood pressure in beta-blocker users. Clin. Pharmacol. Ther. 85(1), 45-50 (2009).

4 Orkin S, Fisher D, Look AT, Lux S, Ginsburg D, Nathan D. Oncology of Infancy and Childhood. Saunders Elsevier, PA, USA (2009).

5 Treleaven J, Barrett AJ. Hematopoietic Stem Cell Transplantation in Clinical Practice. Churchill Livingstone Elsevier, Edinburgh, UK (2009).

6 Pasquini MC, Wang Z. Current use and outcome of hematopoietic stem cell transplantation: CIBMTR Summary Slides. (2013). www.cibmtr.org

7 Mullighan C, Petersdorf E, Davies SM, Dipersio J. From trees to the forest: genes to genomics. Biol. Blood Marrow Transplant. 17(1 Suppl.), S52-S57 (2011)

8 Chien JW, Zhao LP, Storer B et al. Improving hematopoietic cell transplant outcomes in a new era of genomic research. Biol. Blood Marrow Transplant. 15(1 Suppl.), 42-45 (2009).

9 Lupu M, Storb R. Five decades of progress in haematopoietic cell transplantation based on the preclinical canine model. Vet. Comp. Oncol. 5(1), 14-30 (2007).

10 Storb R, Epstein RB, Rudolph RH, Thomas ED. Allogeneic canin bone marrow transplantation following cyclophosphamide. Transplantation 7(5), 378-386 (1969).
11 Australian Institute of Health and Welfare. Cancer survival and prevalence in Australia: period estimates from 1982 to 2010. Cancer Series no. 69. (2012).

www.aihw.gov.au

12 Edwards BK, Noone AM, Mariotto AB et al. Annual Report to the Nation on the status of cancer, 1975-2010, featuring prevalence of comorbidity and impact on survival among persons with lung, colorectal, breast, or prostate cancer. Cancer 120 (9), 1290-1314 (2014).

13 Table of Pharmacogenomic Biomarkers in Drug Labeling. www.fda.gov

14 HUGO Gene Nomenclature Committee. www.genenames.org

15 Deeg HJ. How I treat refractory acute GVHD. Blood 109(10), 4119-4126 (2007).

16 Ashbee HR, Gilleece MH. Has the era of individualised medicine arrived for antifungals? A review of antifungal pharmacogenomics. Bone Marrow Transplant. 47(7), 881-894 (2012).

17 Mullighan CG, Bardy PG. New directions in the genomics of allogeneic hematopoietic stem cell transplantation. Biol. Blood Marrow Transplant. 13(2), 127-144 (2007).

18 Rocha V, Porcher R, Fernandes JF et al. Association of drug metabolism gene polymorphisms with toxicities, graft-versushost disease and survival after HLA-identical sibling hematopoietic stem cell transplantation for patients with leukemia. Leukemia 23(3), 545-556 (2009).

-. Rocha clinical outcomes post transplant, pharmacokinetics and genotype for a number of drug metabolising enzymes for multi agent preparative regimens.

19 Evans WE, Relling MV. Moving towards individualized medicine with pharmacogenomics. Nature 429 (6990), 464-468 (2004).
20 Chabner BA, Longo DL. Cancer Chemotherapy and Biotherapy. Lippincott Williams \& Wilkins, PA, USA. (2011).

21 Galaup A, Paci A. Pharmacology of dimethanesulfonate alkylating agents: busulfan and treosulfan. Expert Opin. Drug Met. 9(3), 333-347 (2013).

22 Andersson BS, Madden T, Tran HT et al. Acute safety and pharmacokinetics of intravenous busulfan when used with oral busulfan and cyclophosphamide as pretransplantation conditioning therapy: a Phase I study. Biol. Blood Marrow Transplant. 6(5), 548-554 (2000).

23 Bartelink IH, Bredius RG, Ververs TT et al. Once-daily intravenous busulfan with therapeutic drug monitoring compared with conventional oral busulfan improves survival and engraftment in children undergoing allogeneic stem cell transplantation. Biol. Blood Marrow Transplant. 14(1), 88-98 (2008).

24 Kashyap A, Wingard J, Cagnoni P et al. Intravenous versus oral busulfan as part of a busulfan/cyclophosphamide preparative regimen for allogeneic hematopoietic stem cell transplantation: decreased incidence of hepatic venoocclusive disease (HVOD), HVOD-related mortality, and overall 100-day mortality. Biol. Blood. Marrow Transplant. 8(9), 493-500 (2002).

25 Johnson L, Orchard PJ, Baker KS et al. Glutathione S-transferase A1 genetic variants reduce busulfan clearance in children undergoing hematopoietic cell transplantation. J. Clin. Pharmacol. 48(9), 1052-1062 (2008).

- Johnson et al. were the first to investigate the relationship between pharmacokinetic parameters and glutathione-S-transferase polymorphisms in pediatric patients receiving intravenous busulfan.

26 Vassord C, Lapoumeroulie C, Koumaravelou K, Srivastava A, Krishnamoorthy R. 
Endothelial cells do not express GSTA1: potential relevance to busulfan-mediated endothelial damage during haematopoietic stem cell transplantation. Eur. J. Haematol. 80 (4), 299-302 (2008).

27 Zwaveling J, Press R, Bredium RGM et al. Glutathione $\mathrm{S}$-transferase polymorphisms are not associated with population pharmacokinetic parameters of busulfan in pediatric patients. Ther. Drug Monit. 30, 504-510 (2008).

- Zwaveling et al. largest pediatric cohort analysis of pharmacokinetics, transplant outcomes and glutathione-S-transferase polymorphisms for intravenous busulfan.

28 Mccune JS, Holmberg LA. Busulfan in hematopoietic stem cell transplant setting. Expert Opin. Drug Met. 5(8), 957-969 (2009).

29 Ansari M, Lauzon-Joset JF, Vachon MF et al. Influence of GST gene polymorphisms on busulfan pharmacokinetics in children. Bone Marrow Transplant. 45(2), 261-267 (2010).

30 Elhasid R, Krivoy N, Rowe JM et al. Influence of glutathione S-transferase A1, P1, M1, T1 polymorphisms on oral busulfan pharmacokinetics in children with congenital hemoglobinopathies undergoing hematopoietic stem cell transplantation. Pediatr. Blood Cancer 55(6), 1172-1179 (2010).

31 Gaziev J, Nguyen L, Puozzo C et al. Novel pharmacokinetic behavior of intravenous busulfan in children with thalassemia undergoing hematopoietic stem cell transplantation: a prospective evaluation of pharmacokinetic and pharmacodynamic profile with therapeutic drug monitoring. Blood 115(22), 4597-4604 (2010).

32 Abbasi N, Vadnais B, Knutson JA et al. Pharmacogenetics of intravenous and oral busulfan in hematopoietic cell transplant recipients. J. Clin. Pharmacol. 51(10), 1429-1438 (2011).

33 Kim SD, Lee JH, Hur EH et al. Influence of GST gene polymorphisms on the clearance of intravenous busulfan in adult patients undergoing hematopoietic cell transplantation. Biol. Blood Marrow Transplant. 17(8), 1222-1230 (2011).

34 Ten Brink MH, Wessels JA, Den Hartigh J et al. Effect of genetic polymorphisms in genes encoding GST isoenzymes on BU pharmacokinetics in adult patients undergoing hematopoietic SCT. Bone Marrow Transplant. 47(2), 190-195 (2012).

35 Hassan M, Andersson BS. Role of pharmacogenetics in busulfan/ cyclophosphamide conditioning therapy prior to hematopoietic stem cell transplantation. Pharmacogenomics 14(1), 75-87 (2013).

36 Bonifazi F, Storci G, Bandini G et al. Glutathione transferase-A2 S112T polymorphism predicts survival, transplantrelated mortality, busulfan and bilirubin blood levels after allogeneic stem cell transplantation. Haematologica 99(1), 172-179 (2014).

37 Mccune JS, Baker KS, Blough DK et al. Variation in prescribing patterns and therapeutic drug monitoring of intravenous busulfan in pediatric hematopoietic cell transplant recipients. J. Clin. Pharmacol. 53(3), 264-275 (2013).

38 Kusama M, Kubota T, Matsukura Y et al. Influence of glutathione S-transferase A1 polymorphism on the pharmacokinetics of busulfan. Clin. Chim. Acta. 368(1-2), 93-98 (2006).

- Kusama et al. were the first to demonstrate a relationship between pharmacokinetic parameters and glutathione-S-transferase polymorphisms in adults receiving oral busulfan.

39 Srivastava A, Poonkuzhali B, Shaji RV et al. Glutathione S-transferase M1 polymorphism: a risk factor for hepatic venoocclusive disease in bone marrow transplantation. Blood 104(5), 1574-1577 (2004).

- Srivastasta et al. were the first to describe a relationship between hematopoietic stem cell transplant clinical outcomes and genotype pediatric patients receiving oral busulfan.

40 Kim MS, Kang HJ, Park HJ et al. Development of multiplex PCR method for the analysis of glutathione $S$-transferase polymorphism. Mol. Diagn. Ther. 15(5), 285-229 (2011).

41 Vassal G, Deroussent A, Hartmann O et al. Dose-dependent neurotoxicity of high-dose busulfan in children: a clinical and pharmacological study. Cancer Res. 50(19), 6203-6207 (1990).

42 Kim IW, Yun HY, Choi B et al. Population pharmacokinetics analysis of cyclophosphamide with genetic effects in patients undergoing hematopoietic stem cell transplantation. Eur. J. Clin. Pharmacol. 69(8), 1543-1551 (2013).

43 Gruhn B, Kloppner N, Pfaffendorf-Regler N et al. Toll-like 4 receptor variant, Asp299Gly, and reduced risk of hemorrhagic cystitis after hematopoietic stem cell transplantation. Biol. Blood Marrow Transplant. 18(6), 958-963 (2012).

- Gruhn et al. were the first to demonstrate the relationship between hemorrhagic cystitis post-transplant and Toll-like receptor 4 single nucleotide polymorphisms.

44 Kuhne A, Sezer O, Heider U et al. Population pharmacokinetics of melphalan and glutathione S-transferase polymorphisms in relation to side effects. Clin. Pharmacol. Ther. 83(5), 749-757 (2008).

45 Ekhart C, Doodeman VD, Rodenhuis S, Smits PH, Beijnen JH, Huitema AD. Polymorphisms of drug-metabolizing enzymes (GST, CYP2B6 and CYP3A) affect the pharmacokinetics of thiotepa and tepa. Br. J. Clin. Pharmacol. 67(1), 50-60 (2009).

- Ekhart et al. only pharmacogenomic analysis published for thio-TEPA use in stem cell transplant.

46 Sanacore M, Bashey A, Sizemore CA et al. Calcineurin inhibitor-free GVHD Prophylaxis with post-transplant cyclophosphamide and brief-course sirolimus results in low rates of non-relapse mortality and chronic GVHD following matched related and unrelated donor peripheral blood stem cell transplantation (PBSCT). Blood 122(21), 923-923 (2013).

47 Mcdonald GB, Slattery JT, Bouvier ME et al. Cyclophosphamide metabolism, liver toxicity, and mortality following hematopoietic stem cell transplantation. Blood 101(5), $2043-$ 2048 (2003).

48 Rodriguez-Antona C, Ingelman-Sundberg M. Cytochrome P450 pharmacogenetics and cancer. Oncogene 25(11), 1679-1691 (2006).

49 Gervasini G, Vagace JM. Impact of genetic polymorphisms on chemotherapy toxicity in childhood acute lymphoblastic leukemia. Front. Genet. 3, 249 (2012).

50 Huang RS, Duan S, Bleibel WK et al. A genome-wide approach to identify genetic variants that contribute to etoposide-induced cytotoxicity. Proc. Natl Acad. Sci. USA 104(23), 9758-9763 (2007).

51 Bleibel WK, Duan S, Huang RS et al. Identification of genomic regions contributing to etoposide-induced cytotoxicity. Hum. Genet. 125(2), 173-180 (2009).

52 Bailey CM, Anderson KS. A mechanistic view of human mitochondrial DNA polymerase gamma: providing insight into drug toxicity and mitochondrial disease. Biochim. Biophys. Acta 1804(5), 1213-1222 (2010).

53 Rossi D, Bruscaggin A, Spina V et al. Mutations of the SF3B1 splicing factor in chronic lymphocytic leukemia: association with progression and fludarabine-refractoriness. Blood 118(26), 6904-6908 (2011).

54 Mould D, Baumann A, Kuhlmann J et al. Population pharmacokinetics- 
pharmacodynamics of alemtuzumab (Campath $\left.{ }^{\circledR}\right)$ in patients with chronic lymphocytic leukaemia and its link to treatment response. Brit. J. Clin. Pharmaco. 64(3), 278-291 (2007).

Qui F, He XJ, Sun YX, Li-Ling J, Zhao LM. Influence of $A B C B 1, C Y P 3 A 4^{*} 18 B$ and $C Y P 3 A 5^{*} 3$ polymorphisms on cyclosporine $\mathrm{A}$ pharmacokinetics in bone marrow transplant recipients. Pharmacol. Rep. 63, 815-825 (2011).

56 Cao W, Xiao H, Lai X et al. Genetic variations in the mycophenolate mofetil target enzyme are associated with acute GVHD risk after related and unrelated hematopoietic cell transplantation. Biol. Blood Marrow Transplant. 18(2), 273-279 (2012).
57 Kim I, Keam B, Lee KH et al. Glutathione S-transferase A1 polymorphisms and acute graft-vs.-host disease in HLA-matched sibling allogeneic hematopoietic stem cell transplantation. Clin. Transplant. 21(2), 207-213 (2007).

58 Murphy N, Diviney M, Szer J et al. Donor methylenetetrahydrofolate reductase genotype is associated with graft-versus-host disease in hematopoietic stem cell transplant patients treated with methotrexate. Bone Marrow Transplant. 37(8), 773-779 (2006).

59 Robien K, Schubert MM, Bruemmer B, Lloid ME, Potter JD, Ulrich CM. Predictors of oral mucositis in patients receiving hematopoietic cell transplants for chronic myelogenous leukemia. J. Clin. Oncol. 22(7), 1268-1275 (2004).

60 Kim IW, Yun HY, Choi B et al. ABCB1 C3435T genetic polymorphism on population pharmacokinetics of methotrexate after hematopoietic stem cell transplantation in Korean patients: a prospective analysis. Clin. Ther. 34(8), 1816-1826 (2012).

61 Ten Brink MH, Swen JJ, Bohringer $S$ et al. Exploratory analysis of 1936 SNPs in ADME genes for association with busulfan clearance in adult hematopoietic stem cell recipients. Pharmacogenet Genomics 23(12), 675-683 (2013). 